\title{
Risk Evaluation of Big Sport Events Based on Neural Networks
}

\author{
Yong-Sheng WANG ${ }^{1, a}$,Quan-Ying $\mathrm{LI}^{2, \text { a }}$ \\ ${ }^{1}$ Department of Physical education, North-west University, Xi'an, 710069, China \\ ${ }^{2} \mathrm{Xi}$ 'an electric power college, Xi'an, 710032, China \\ a724350372@qq.com
}

Keywords: Neural networks, Large-scale sporting events, Risk evaluation

\begin{abstract}
In this paper, we conclude the risk of big sport events from 17 factors of four aspects using literature analysis and expert interviews. We use BP neural network to evaluate the risk of the big events. Using Matlab to simulation the models, we provide a novel method for the risk-assessment of big sport events.
\end{abstract}

\section{Introduction}

The risk of big sports events usually bring very serious consequences. It not only generate great influence to the competitions, but also leads to great loss to the participants[1]. Therefore, in the race operation, we need to take effective measures to do risk assessment and reduce the risk in order to reduce the loss and promote the successful processing of the races[2]. Also it can further promote the industrialization and market-oriented development of sports. Therefore, for big sports events, risk assessment has very important significance.

BP neural network consists of an input layer, a hidden layer and output layer. Its dissemination and training process include two steps, the forward error correction and convey[3]. Error back makes error analysis continually approach the preset error. The training of BP neural network can end when the fitting degree satisfies the decision makers or the trails for studying reach the set value[4]. At this moment, the output of the system is the target of the evaluation. In this paper, we develop an evaluation of the risks of big sport events based on the BP neural network risk-assessment models. We set the results of case study as the input of the model and the Matlab software was used for the simulation. Using our models we can predict the level of the sports event and offer a objective indicator for government and organizers. Also, it can offer us evident for the big sport events.

\section{Risk Indicators Analysis for Big Sport Events}

The occurrence of the risks of big sports events have a certain incentives, which are those various primary cause or risk causing big sports events, as well as the risk of a change in the results of those risk events, objective factors. Objective factors are the risks refer to big sports events of external environment factors. In some external environment of a sporting events, it will be subject to any external environment influenced by many factors, such as natural, political, economic, cultural, legal and other factors. Single or comprehensive changes in various factors make the development of the results in a variety of events manifested in unexpected ways, which are not the people's subjective will and cannot be sure and grasp. The results of subjective factors is the risk that in organizational behavior from the activities of the event itself implied uncertainty caused. A large 
organizational activity includes a variety of sporting events. Because of the limitations of the subjective understanding of information collected on the limited nature of the development of things, grasping the basic law of universal connection between the bound has some limitations. It is difficult to predict in a timely manner of the development trend of things, so that in itself has a certain risk. Therefore, these factors tend to lead to a potential incentive for the risks of big sports events.

\section{Development of a Risk Evaluation System for Big Sport Events}

During major sporting events there might be encountered equipment failure, injured athletes, referees injustice and a series of unexpected problems. Considering the competition venues, schedule and timing and transportation, information and communications, security facilities, weather conditions and cultural facilities and other components of a dynamic system, any part of a problem, it will seriously affect the smooth conduct of sporting events. According to tournament transport systems activities, different objects can be divided into organizational risk, management risk, personnel risk, business risk and environmental risks. Every type of risk can be divided in to various factors, which are shown in Figure 1.

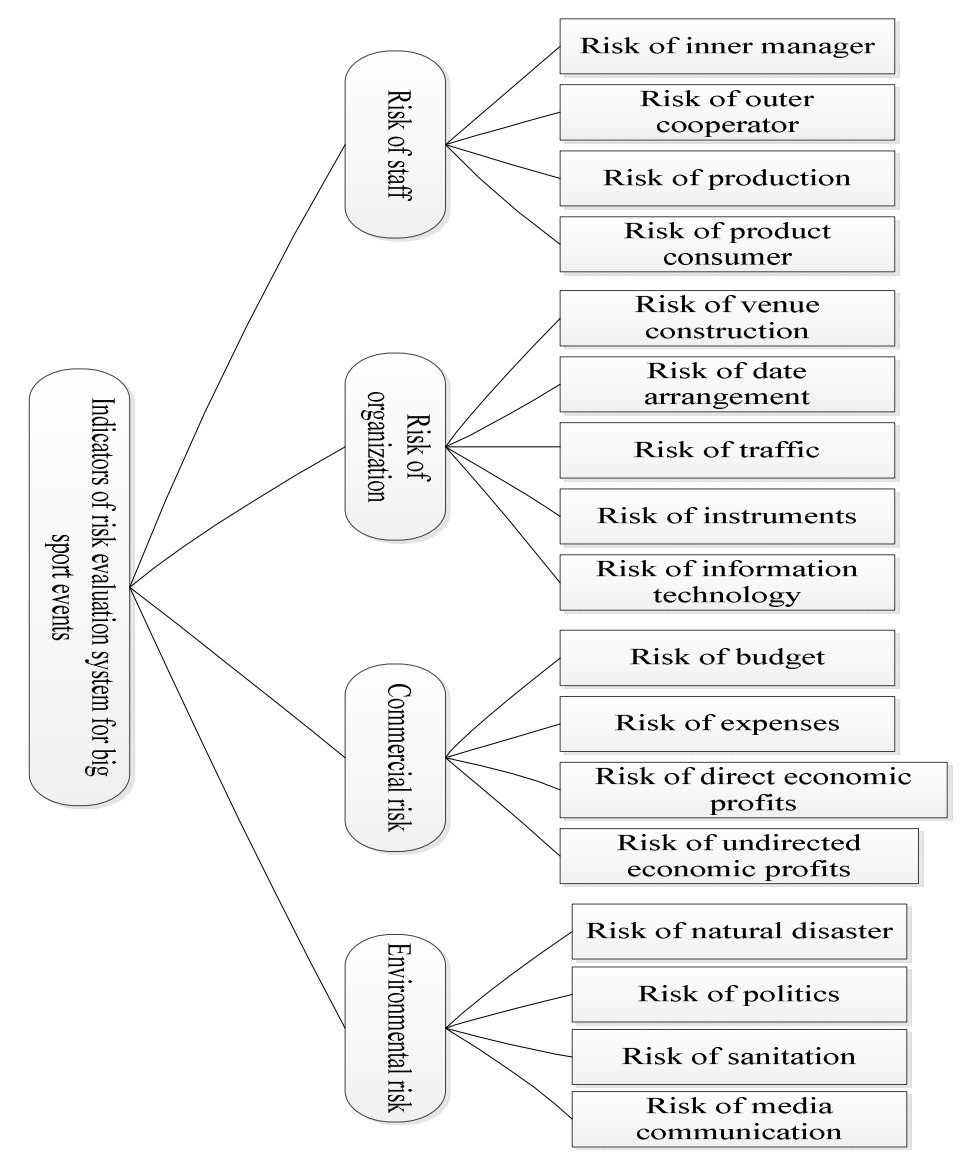

Fig. 1 Indicators of risk evaluation system for big sport events

\section{Risk Evaluation Model Based on BP Neural Network}

\section{Selection of Input Layer}

The input layer of the BP neural network is the risk indicators of the evaluation system for big sport events. This paper selects internal risk manager and external collaborators risk, product risk implementor, consumer product risk, venue construction risk, schedule risk, transportation risk, the 
risk of venues and equipment, information technology, risk, risk budgeting, expenditure risk, the direct economic benefits, the risk of indirect economic benefits, natural disaster risk, political risk, health and safety risks and the risk of transmission media as the BP neural network input vector, which are presented by $\mathrm{X}$ i .

\section{Design of Hidden Layer}

Increase in hidden layer will increase the complexity and training time training BP neural network. Therefore, to speed up the convergence, each node in the hidden layer uses S-type function as the activation function. The output of the BP neural network is:

$$
y=f(n e t)=\left(1+e^{-n e t}\right)^{-1}
$$

wherenet is the input net $=x_{1} w_{1}+x_{2} w_{2}+\cdots+x_{n} w_{n}$

The determination of number of nodes in the hidden layer is a comparatively complex problem. Here we use a defined number of nodes, where $\mathrm{n}$ is the number of input nodes.

\section{Design of Output Layer}

The output of the model is the measurement of the risks of big sport events. Therefore, the node in the output layer is one, which is represented by $\circ$. To satisfy the needs of cluster analysis, the risks are divided into four levels: I level represents the low risk, with the output result $(0,0.25)$; I I level represents that the risk is general, with the output result $(0.25,0.5)$; I I I level represents that the risks are high, with the output result of $(0.5,0.75]$; I V represents the very high risk, with the output result of $(0.75,1)$

According to the discussion above, the model for the risk evaluation of big sport events based on BP neural network is shown as Figure 2.

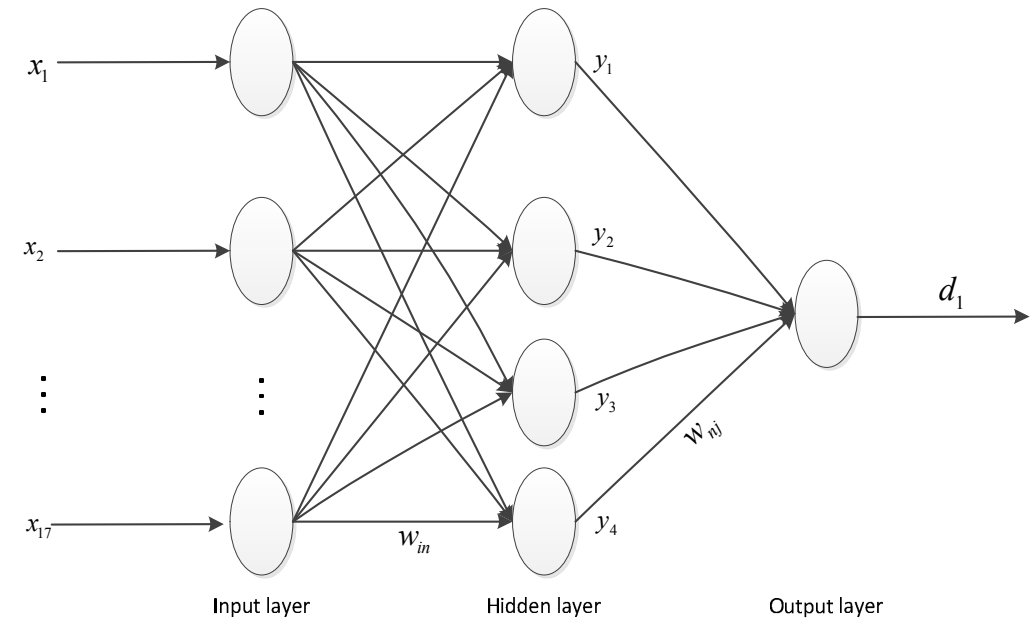

Fig. 2 The model for the risk evaluation of big sport events based on BP neural network

\section{Model Simulation}

\section{Obtain the Input of the Model}

To acquire the sample data of the training, we analyzed the issues of the big sport events in recent 5 years. By describing the risk, we define 17 kinds of risks in 10 sport events as the samples after quantifying the risks in accordance with the issues (which is the input of the model). Results are shown in Table 1. 
Table 1 Model input

\begin{tabular}{lllllllllll}
\hline & P1 & P2 & P3 & P4 & P5 & P6 & P7 & P8 & P9 & P10 \\
\hline$x_{1}$ & 0.8 & 0.9 & 0.7 & 0.5 & 0.5 & 0.5 & 0.5 & 0.5 & 0.6 & 0.6 \\
$x_{2}$ & 0.5 & 0.3 & 0.3 & 0.3 & 0.3 & 0.3 & 0.3 & 0.5 & 0.3 & 0.3 \\
$x_{3}$ & 0.5 & 0.5 & 0.3 & 0.3 & 0.3 & 0.3 & 0.3 & 0.3 & 0.1 & 0.5 \\
$x_{4}$ & 0.3 & 0.1 & 0.5 & 0.3 & 0.3 & 0.3 & 0.3 & 0.5 & 0.5 & 0.3 \\
$x_{5}$ & 0.3 & 0.3 & 0.3 & 0.1 & 0.3 & 0.5 & 0.1 & 0.5 & 0.3 & 0.3 \\
$x_{6}$ & 0.5 & 0.1 & 0.3 & 0.5 & 0.3 & 0.2 & 0.3 & 0.3 & 0.1 & 0.5 \\
$x_{7}$ & 0.1 & 0.5 & 0.3 & 0.1 & 0.3 & 0.2 & 0.3 & 0.5 & 0.1 & 0.3 \\
$x_{8}$ & 0.8 & 0.9 & 0.7 & 0.5 & 0.5 & 0.5 & 0.5 & 0.5 & 0.6 & 0.6 \\
$x_{9}$ & 0.5 & 0.3 & 0.3 & 0.3 & 0.3 & 0.3 & 0.3 & 0.5 & 0.3 & 0.3 \\
$x_{10}$ & 0.5 & 0.5 & 0.3 & 0.3 & 0.3 & 0.3 & 0.7 & 0.3 & 0.1 & 0.5 \\
$x_{11}$ & 0.3 & 0.1 & 0.5 & 0.3 & 0.3 & 0.3 & 0.3 & 0.2 & 0.5 & 0.3 \\
$x_{12}$ & 0.3 & 0.3 & 0.3 & 0.1 & 0.3 & 0.5 & 0.1 & 0.5 & 0.3 & 0.3 \\
$x_{13}$ & 0.5 & 0.1 & 0.3 & 0.5 & 0.3 & 0.2 & 0.3 & 0.3 & 0.1 & 0.5 \\
$x_{14}$ & 0.6 & 0.1 & 0.7 & 0.7 & 0.5 & 0.1 & 0.3 & 0.5 & 0.3 & 0.3 \\
$x_{15}$ & 0.5 & 0.3 & 0.3 & 0.3 & 0.3 & 0.3 & 0.3 & 0.5 & 0.3 & 0.3 \\
$x_{16}$ & 0.7 & 0.5 & 0.3 & 0.3 & 0.3 & 0.3 & 02 & 0.3 & 0.1 & 0.5 \\
$x_{17}$ & 0.3 & 0.4 & 0.5 & 0.4 & 0.3 & 0.3 & 0.3 & 0.5 & 0.5 & 0.5 \\
\hline
\end{tabular}

\section{Training and Simulation Processes of the Model}

(1) Initialize the BP neural network using the newff() function. A feedforward BP network is developed by the initialization as follows:

1) Hidden layer activation function takes tansig function.

2) Output layer activation function uses logsig functions.

3) Training function takes traingd gradient descent function.

4) Learning function uses the default function, which is the learngdm function.

5) Performance function uses the default function, which is the mse function.

6) The calculating precision $\varepsilon$ is defined as 0.0005 .

7) The max learning trails $M$ is defined as 1000.

(2) Randomly selected $k$-th input samples and the corresponding desired output. For example, we choose $\mathrm{P} 1 \sim \mathrm{P} 5$ as the input sample:

$$
x(k)=\left(x_{1}(k), x_{2}(k), \cdots, x_{17}(k)\right) k=1,2, \ldots, 5 .
$$

(3) Calculate the input and output of each neuron in each layer via Matlab. And calculate the partial derivatives of the error function for each neuron output layer.

(4) Calculate the hidden layer output by MATLAB, computing the partial derivatives of the error function hidden layer neurons.

(5) Calculate the overall error.

With the increase of the number of training, analysis of errors continues approximation error preset value. When the decision makers accept the approach that has reached a degree or learning a preset number of times, the algorithm ends. Otherwise, we should select the next learning samples and corresponding desired output and continue the training. 


\section{Model Simulation}

According to the steps above, we simulate the model of Table using Matlab 7.0 simulation platform. The 12 items in Table 1 were inputted into Figure 2. The errors between the output results and the actual results decreased with the increase of training trails. When the training trails reaches the max trails, the errors are lower than the desired errors. Then the simulation ends.

\section{Model Application}

Here we use the model to evaluate the 15th sports meeting in Shanxi Province. The indicators are

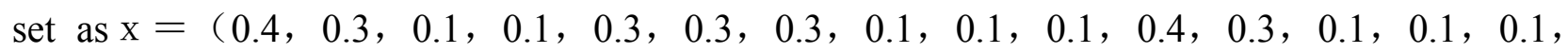
$0.1,0.3$ ). The model in Figure 3 was inputted. After the Matlab simulation, we can obtain the output result of the model 0.383 . We can clearly see that this project is in the risk level of I I

\section{Summary}

In this paper, we propose a novel evaluation model, method and steps for big sport events. According to the indicators of big sport events, we can input this model based on BP neural network and determine the risk level after the simulation results of Matlab simulation.

\section{References}

[1] Jie D, Lixin L,Research on Construction of Risk Assessment System forLarge-scale Sporting Event, Journal of Wuhan Institute of P.E, 41(2007)32-36.

[2] Xiao D, Chunling G, sport law, third ed., Law Press China, Bei Jing, 2006.

[3] XIA Yimin, BIAN Zhangkuo, JI Zhiyong, et al., Development of cutter Head CAD system for compositeEPB shield, Computer Engineering and Applications,48(2012)64-69.

[4] H. Li, W. Leng, Y. Zhou, F. Chen, Z. Xiu, D. Yang, Evaluation models for soil nutrient based on support vector machine and artificial neural networks, The Scientific World Journal, 2014 (2014) 478569. 